\title{
Acquisition and extinction following extended partial reinforcement training under small or large reward
}

\author{
LAWRENCE S. MEYERS and GARY J. ANDERSON \\ California State University, Sacramento, California 95819
}

\begin{abstract}
Forty-one rats were given either 30,90 , or $30050 \%$ partial reinforcement trials to either a 1 - or 15 pellet reward. Following both 30 and 90 trials, large reward led to faster acquisition responding and greater resistance to extinction than small reward. Following 300 trials, small and large reward groups were responding equally rapidly in acquisition, but the small reward group tended to be more resistant to extinction than the large reward group.
\end{abstract}

On the basis of considerable empirical evidence (e.g., Capaldi \& Capaldi, 1970; Capaldi \& Lynch, 1968; Hulse, 1958; Wagner, 1961) it is generally assumed that groups receiving large reward during partial reinforcement training demonstrate greater terminal acquisition performance and greater resistance to extinction than groups receiving small reward during partial reinforcement. These assumptions are incorporated into the contemporary animal learning theories of Amsel (e.g., 1958; 1962) and Capaldi (e.g., 1967).

A recent study by McCain (1970) questions the generality of these reward magnitude effects across all levels of training. McCain reported that although "large-partial" was associated with faster responding and greater resistance to extinction than "small-partial" following 42 acquisition trials, after 138 training trials small and large partial reinforcement groups had comparable terminal acquisition speeds and were equally resistant to extinction.

Since McCain's (1970) findings apparently contrast with currently available data, a more extensive investigation of the roles of reinforcement magnitude and levels of training appears warranted. In the present experiment animals received either 30,90 , or 300 partial reinforcement trials under either small or large reward and were then extinguished.

\section{METHOD}

The 42 male Sprague-Dawley rats bred at Sacramento State were about 105 days old at the beginning of the experiment, and were randomly assigned to six groups of seven subjects each. One animal scheduled for 300 trials under large reward died during training and its data were entirely disregarded. The apparatus used was a standard Gerbrand's Model C operant chamber equipped with a Scientific Prototype (RL2-1) retractable bar.

Three levels of $50 \%$ partial reinforcement acquisition training (30, 90, and 300 trials) were factorially combined with two levels of reward ( 1 and $15.045 \mathrm{~g}$ pellets). All animals were placed on a deprivation schedule on Day 1 involving $1 \mathrm{~h}$ free access to Lab Chow every $23 \mathrm{~h}$. On Day 15 those subjects to

This paper was presented to the meeting of the Western Psychological Association, Anaheim, California, April, 1973. F.eprint requests should be sent to Lawrence $S$. Meyers, Department of Psychology, California State University, Sacramento, California 95819 . receive 300 trials were shaped to a criterion of 10 barpresses under one pellet reinforcement and on Day 16 they received 10 trials of $100 \%$ reinforcement at their scheduled reward. From Day 17 through Day 46, 300) partial reinforcement trials were administered at a rate of ten trials each daily session. On Days 36 and 42 , the two pretraining and the appropriate number of training sessions were instituted for subjects receiving 90 and 30 acquisition trials, respectively. A maximum of three rewarded and three nonrewarded trials occurred in succession on any one training day. Further, beginning from Day 1, all subjects in all groups were given equal handling regardless of their daily experimental routine. During training, the bar was retracted for a period of $90 \mathrm{sec}$ following each barpress. The latency from the insertion of the bar into the box until a response occurred was recorded.

All animals entered extinction on Day 47, at a rate of 10 trials per day. If a barpress was not made within $5 \mathrm{~min}$ after the appearance of the bar, the bar was retracted and presented again $90 \mathrm{sec}$ later. Two successive 5-min trials ended the session for the subject, and he was assigned the maximum score for the remaining trials of the day. Two terminated sessions resulted in the retirement of the animal and he was assigned the maximum score for all remaining sessions. The retirement of three animals in a group was the criterion for terminating both that group and the other group which received a comparable amount of acquisition training.

\section{RESULTS}

All latency measures were transformed to speed scores. The acquisition data are presented in Figure 1, with speed multiplied by 1000 and plotted over training sessions. For the 30-trials group, the trend of faster responding under large compared to small reward was not a reliable one, i.e., neither the main groups effect nor the Groups by Acquisition Sessions interaction reached significance, Fs $<1$. For animals given 90 trials, those responding under large reward improved at a more rapid rate than those pressing for small reward as indicated by the reliable Groups by Acquisition Sessions interaction $\left[\mathrm{F}(8 / 96)=3.11, \mathrm{p}<.05, \eta^{2}=.13\right]$; the overall group difference was not significant $[F(1 / 12)=4.57, p>.05]$. On the final day of training no group difference was observed, $F<1$. Although similar differences were found after 90 trials for the extended training groups, by the completion of their 300 training trials no performance differences were associated with reward magnitude, $\mathrm{F}<1$. 

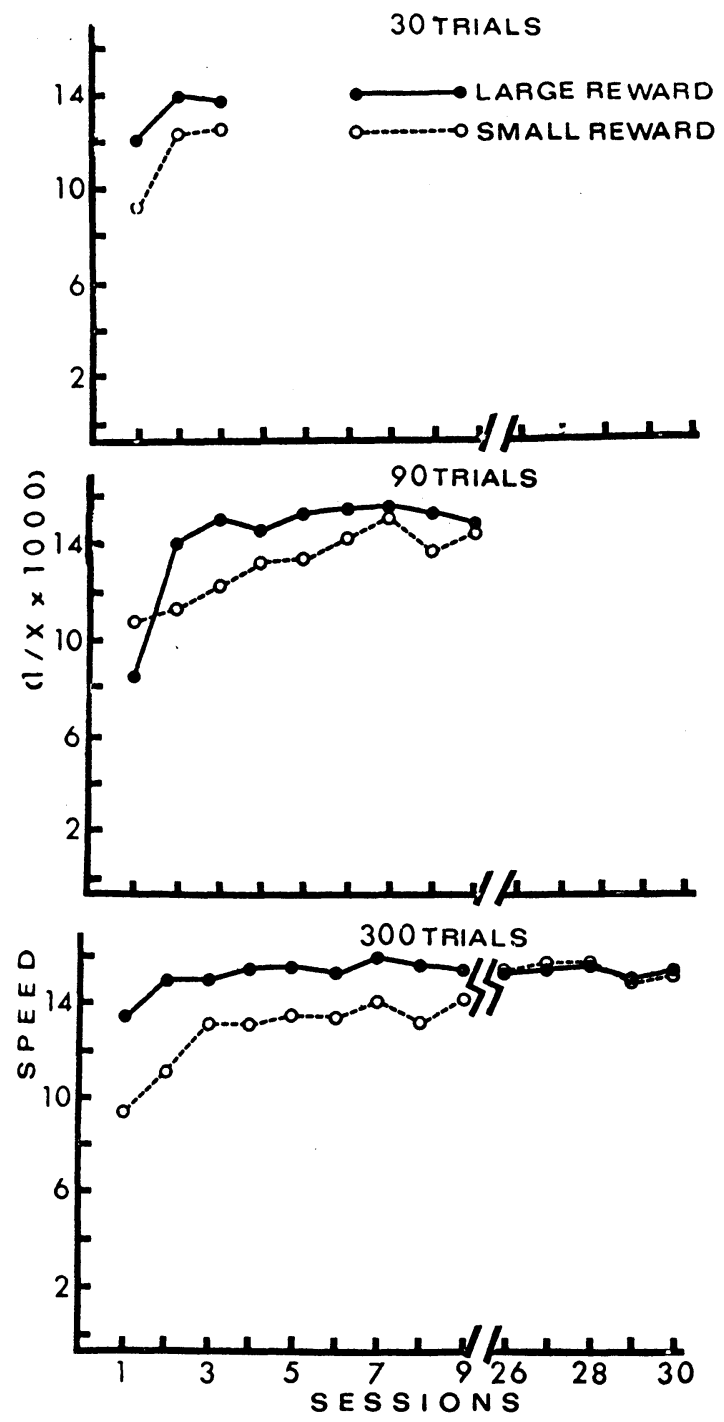

Figure 1. Acquisition over sessions of 10 trials each.

The extinction performance of each of the groups is presented in Figure 2. Recall that the procedure dictated that the two groups at each acquisition level be terminated together; on this basis the 30-, 90-, and 300-trials groups reached the extinction criterion after 70,100 , and 130 trials, respectively. For both the 30and 90-trials groups extinction differences appeared during the first session and were maintained through most of extinction. In both the 30- and 90-trials conditions, large reward produced greater resistance to extinction than small reward $\left[\mathrm{F}=5.91, \eta^{2}=.26\right.$ and $\mathrm{F}=7.14, \eta^{2}=.31$, respectively $]$, with $\mathrm{dfs}=1 / 12$ and ps $<.05$. In both analyses the Groups by Extinction Sessions interactions were not significant, Fs $<1$.

Following 300 trials of acquisition, the small reward group showed a tendency to be less resistant to extinction than the large reward group during the first six sessions; following the seventh session speed of pressing for the large reward group sharply decreased relative to the constant but slower response decrement observed for the small reward subjects. However, an unequal $\mathrm{N}$ analysis of variance encompassing all extinction sessions yielded neither a groups effect nor a Groups by Extinction Sessions interaction, Fs $<1$. Two further analyses, conducted on a post hoc basis, suggested that while no differences existed during the initial 30 extinction trials, $\mathrm{F}<1$, the small reward group was more resistant to extinction than the large reward group over the final 30 extinction trials $[\mathrm{F}(1 / 11)=6.63$, $\mathrm{p}<.05$ ]

\section{DISCUSSION}

The present results confirm and extend the suggestions made by McCain (1970), and taken together with McCain's findings provide a somewhat detailed description of partial reinforcement

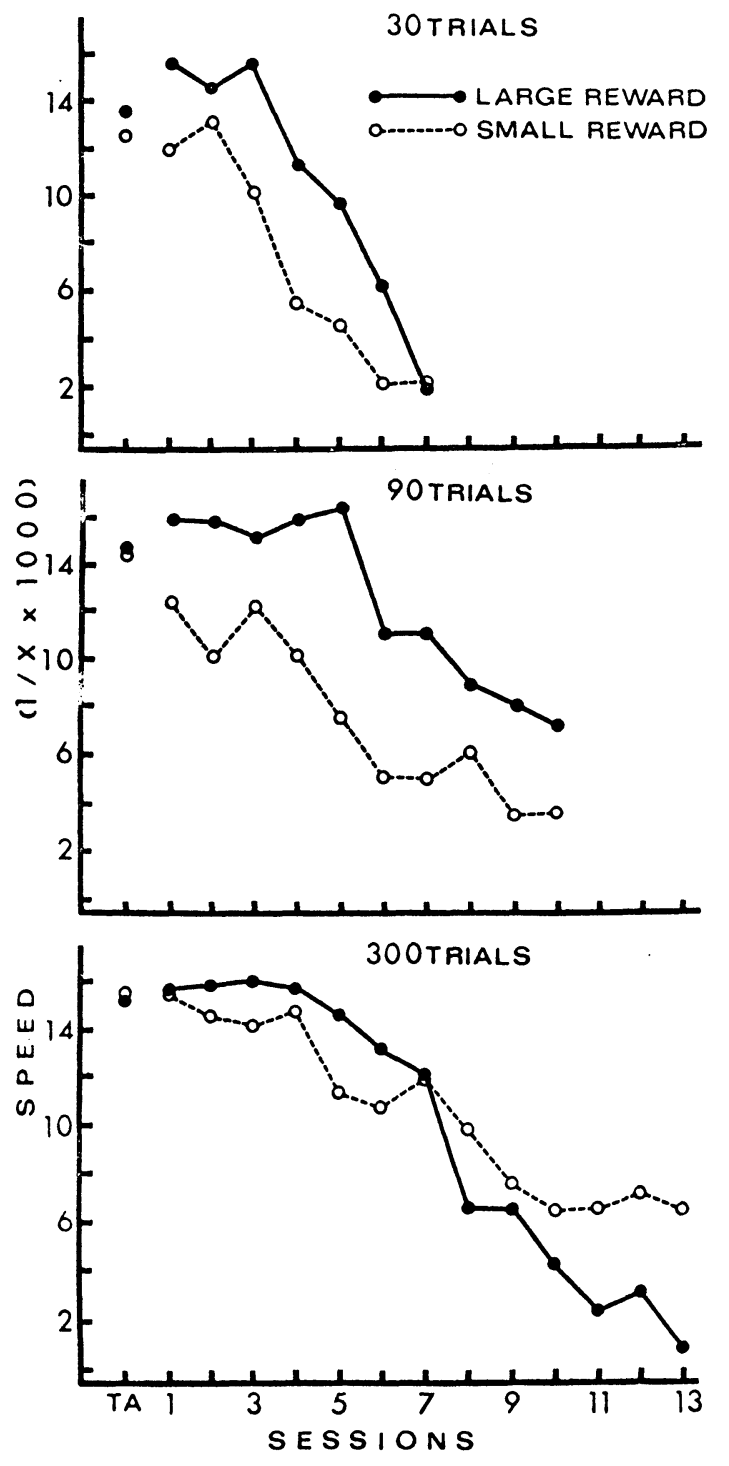

Figure 2. Terminal acquisition (TA) and extinction over sessions of 10 trials each. 
effects. First, it appears that reward magnitude affects the rate at which acquisition asymptote is reached but does not determine asimptotic performance level. Second, the function relating resistance to extinction to magnitude of partial reinforcement is complex and must include a level of training parameter. Specifically, it appears that after limited training large reward is associated with greater resistance to extinction than small reward. that after the moderate amount of training given by McCain (1970), large and small reward do not differentially affect extinction performance, and that after extended acquisition training small reward may tend to produce greater persistence during extinction than large reward.

This description is not inconsistent with the larger body of literature on the problem, given that the available data are considered instances of limited training effects. Such a consideration is reasonable when previously used levels of training are examined. Capaldi and Capaldi (1970), for example. used 18 acquisition trials, Capaldi and Lynch (1968) used 22 trials, Hulse (1958) used 24 trials, and Wagner (1961) used 60 trials as his maximum. Although such cross-experimental comparisons are tenuous here, especially since these studies, as well as McCain's, (1970), dealt with locomotor behavior, it can minimally be said that extended acquisition training was not provided.

Taken together with McCain's (1970) results, the present data bear on the theories of Amsel $(1958 ; 1962)$ and Capaldi (1967). The postulation of conditioned frustration (Amsel, 1958;1962) is used not only to explain the partial reinforcement acquisition effect, but differences in the amount of conditioned frustration based on reward magnitude are also used to account for acquisition and extinction differences between small and large magnitudes of partial reinforcement groups following somewhat limited amounts of training (e.g., Wagner, 1961). The present data would lead to the implication that small compared to large reward following extended acquisition produces comparable amounts of conditioned frustration. The tendency toward more rapid extinction of the large reward compared to the small reward group following extended acquisition could be attributed to a greater magnitude of primary frustration presumably aroused during extinction for the former group.

Capaldi's (1967) model, reflecting data collected under limited or moderate training conditions, assumes that reward maynitude directly determines associative strength. The comparable asymptotic performance presently observed between small and large partial reinforcement groups suggests that a comparable amount of associative strength is achieved under all reward levels, but at different growth rates for each reward magnitude. Since this growth rate appears to be low for small reward conditions the implication is that differences in associative strength are most evident during limited training. Capaldi's analyses of partial reinforcement effects, masnitude of reward effects, and the variety of theoretical treatments of other related phenomena therefore retain their predictive value for limited and moderate amounts of training.
The assumption of equal maxima of associative strength for all levels of reward magnitude, if incorporated into Capaldi's (1967) model, help to explain the extinction performance observed here following extended training. Assuming a comparable sequential structure of the acquisition schedules, then associative strength to each stimulus which was conditioned would be equal. Since one group would have associative strength to the memory of small reward and the other would have associative strength to the memory of large reward, and since greater generalized associative strength to extinction related stimuli would be derived from the small rather than the large reward stimulus set, it directly follows that small-partial would produce greater resistance to extinction than large-partial reinforcement. In fact, given the considerable amount of associative strength resulting from extended practice. one would further predict that extinction differences favoring the small reward group should emerge relatively late in extinction; that is, both groups benefit from generalized associative strength during early extinction, but the small reward group will still be responding to extinction stimuli which have accrued some generalized associative strength long after that no longer holds true for the large reward group. For example, the stimulus set corresponding to the memory of 120 extinction trials will have greater generalized associative strength for the small-partial group than for the large-partial group, a difference substantially greater than that for the stimulus set corresponding to only 40 extinction trials.

\section{REFERENCES}

Amsel, A. The role of frustrative nonreward in noncontinuous reward situations. Psy cholgical Bulletin, 1958, 55, 102-119.

Amsel, A. Frustrative nonreward in partial reinforcement and discrimination learning: Some recent history and a theoretical extension. Psy chological Review, 1962, 69, 306-328.

Capaldi, E. J. A sequential hypothesis of instrumental learning. In K, W. Spence and J. T. Spence (Eds.), The psychology of learning and motivation. New York: Academic Press, 1967.

Capaldi, E. J., \& Capaldi, E. D. Magnitude of partial reward, irregular reward schedules, and a 24-hour ITI. Journal of Comparative and Physiological Psychology, 1970, 72 , 203-209.

Capaldi, E. J., \& Lynch, A. D. Magnitude of partial reward and resistance to extinction. Journal of Comparative and Phy siological Psychology, 1968, 65, 179-181.

Hulse, S. H., Jr. Amount and percentage of reinforcement and duration of goal box confinement in conditioning and extinction. Journal of Experimental Psychology, 1958, 56 48-57.

McCain, G. Reward magnitude and instrumental responses: Consistent and partial reward. Psychonomic Science, 1970. 19, 139-141.

Wagner, A. R. Effects of amount and percentage of reinforcement and number of acquisition trials on conditioning and extinction. Journal of Experimenta Psvchology, 1961,63, 234-242.

(Received for publication May 12, 1975.) 